\title{
Magnetic Field Lines, Hamiltonian Dynamics, and Nontwist Systems
}

\author{
P.J. Morrison \\ Department of Physics and Institute for Fusion Studies, University of Texas at Austin, \\ Austin, Texas 78712, U.S.A.
}

\begin{abstract}
Magnetic field lines typically do not behave as described in the symmetrical situations treated in conventional physics textbooks. Instead, they behave in a chaotic manner; in fact, magnetic field lines are trajectories of Hamiltonian systems. Consequently the quest for fusion energy has interwoven, for 50 years, the study of magnetic field configurations and Hamiltonian systems theory. The manner in which invariant tori break-up in symplectic twist maps, maps that embody one and a half degree-of-freedom Hamiltonian systems in general and describe magnetic field lines in tokamaks in particular, will be reviewed, including symmetry methods for finding periodic orbits and Greene's residue criterion. In nontwist maps, which describe e.g. reverse shear tokamaks and zonal flows in geophysical fluid dynamics, a new theory is required for describing tori break-up. The new theory is discussed and comments about renormalization are made.
\end{abstract}

PACS numbers: 03.20.+i, 47.20.Tg, 52.55.-s 


\section{INTRODUCTION}

Classical mechanics is a very old discipline; depending on where one selects $t=0$, it is at least 100's of years old. It may come as a surprise that important and substantial progress has been made in this field in the last 50 years. In particular, the elementary problem of how a swing behaves or the essentially equivalent problem of explaining the behavior of bundles of closed magnetic field lines when symmetry is broken have been solved. Both of these systems possess nonlinearity and periodicity, and they are, among other things, the subject matter of this talk.

Because this is the APS Centennial meeting it seems appropriate to say a little about the progress that has transpired in this field in the past 100 years. At the turn of the centry Poincaré made impressive discoveries. For systems with two degrees of freedom (two canonical coordinates and two momenta) he introduced the idea that Hamiltonian systems are area preserving maps of planar regions. He of course did much more than this, he worked on the existence of invariant surfaces (tori) and conjectured about their destruction, work that was completed by G. D. Birkhoff. To many he is considered to be the father of the field of topology. However, in spite of the impressive discoveries by Poincaré, Birkhoff, and others in the first half of the twentieth century, the more impressive progress has been made in the second half; namely, the proof of the celebrated KAM theorem, which gives meaning to perturbation theory, the discovery of Smale horseshoes, which gives meaning to the notion of chaos, the clear understanding of the failure of perturbation theory and the nonexistence of action-angle variables. Because of the work of Greene and later researchers, we now understand how invariant tori break and we have a theory of renormalization in Hamiltonian systems. In addition, much progress has been made in understanding transport in Hamiltonian phase space, a major concern of fusion physics, but I will not dwell much on this topic.

In the first half of the twentieth century classical mechanics was a relatively unfashionable discipline. This was the age of modern physics and the parallel development of functional 
analysis in mathematics. Few researchers thought seriously about classical mechanics. In the second half of the twentieth century the quest to achieve energy by controlling fusion reactions by containing hot plasma in magnetic bottles was begun, and impressive progress in this endeavor also was (and is being) made. It is my belief that there is a causal relationship between the progress in fusion and that in classical mechanics. The quest for fusion instigated a renewal in the study of classical mechanics, and indeed plasma physicists have made important contributions to classical mechanics. This is an example of the not uncommon scenario of scientific advancement, where practical and fundamental progress are made handin-hand.

In fusion physics one is interested in containing charged particles and because the particle concentrations of interest are typically small compared to the quantum concentration, particles can be described classically. Thus obviously one is led to the study of classical trajectories in electric and magnetic fields. But because particles follow magnetic field lines to leading order, one needs to understand their nature. In my experience, the typical physicist outside of plasma physics does not have a good feeling for the nature of magnetic field lines. Few know that a single field line can densely fill a surface or wander around forever in a bounded region of space without closing. This is because text books only discuss cases with symmetry. I do not know of a single electricity and magnetism text that treats magnetic field lines seriously. The incorrect belief that $\nabla \cdot \boldsymbol{B}=0$ implies field lines are either closed or go to infinity, surprisingly still exists. Another thing that is not widely known is that the equations that describe magnetic field lines are in fact a Hamiltonian system. Since Hamiltonian trajectories are typically chaotic, the same is true of magnetic field lines.

So I have two main goals:

(i) to desribe some of the progress in classsical mechanics in the last 50 years and to give a sense for why contributions are assoiated with plasma physicists and

(ii) to describe some recent work of my own in collaboration with John Greene and Diego del-Castillo-Negrete ${ }^{1}$ on how invariant tori break in systems that violate the so-called 


\section{twist condition.}

In Sec. II, it is described how both Hamiltonian systems and magnetic field line systems, particularly those of fusion devices, are area preserving maps. Also, ways of casting the equations for field lines in Hamiltonian form are presented. In Sec. III a bit of Hamiltonian dynamics lore is described (e.g. periodic orbits, the rotation number or safety factor, the standard map), but mainly the twist condition is described, an important property possessed by the most studied Hamiltonian systems. Section IV describes systems that violate the twist condition, and here the nontwist map, a prototype map for nontwist behavior, is discussed. Also in this section it is shown how transport in zonal flows, nonmonotonic $q$ profiles, and other systems are nontwist Hamiltonian systems. In Sec. V reconnection in nontwist systems is briefly considered. Here it is noted that island chains come in two types and that this gives rise to a complicated arrangement of periodic orbits. The main problem, when and how do tori break in nontwist systems, is described in Sec. VI. Before describing our results on nontwist systems, we first review Greene's calculation for twist maps and describe an associated procedure for finding periodic orbits. In Sec. VII a few comments about renormalization are made before we summarize in Sec. VIII.

\section{MAGNETIC FIELD LINES ARE HAMILTONIAN TRAJECTORIES}

Let us jump back about 100 years and discuss a result of Poincaré. Consider a Hamiltonian system with two degrees of freedom:

$$
\dot{q}^{i}=\frac{\partial H}{\partial p_{i}}, \quad \dot{p}_{i}=\frac{\partial H}{\partial q^{i}}, \quad i=1,2
$$

where $H=H(q, p)$ does not have explicit dependence upon time. Because it is not possible to visualize the 4-dimensional phase space, we set the Hamiltonian equal to some constant value, say $E$, and plot trajectories in a 3-dimensional space. If the equation $H=E$ can be solved for $p_{2}=p_{2}\left(q_{1}, p_{1}, q_{2}, E\right)$, which is usually the case, then a trajectory can be

plotted in the space with axes $q_{1}, p_{1}, q_{2}$ as depicted in Fig. 1. If the trajectory returns to 
a neighborhood of the initial condition, which must be the case if the energy surface is bounded, then Eqs. (1) define a return map. In appropriate coordinates this map is an area preserving map of a planar region (see e.g. Ref. 2, pp. 151-155). An example of this is shown in Fig. 1, where a trajectory originating in the $q_{1}-p_{1}$ plane returns at a later time to $q_{2}=0$. Near this trajectory there is (at least) a little disk in the plane of trajectories that return, and thus Eqs. (1) define an area preserving map of at least a disk of the $q_{1}-p_{1}$ plane to itself.

Because the trajectories in the disk return to $q_{2}=0$ at different times, it is natural to attempt to find a set of coordinates in which all the trajectories return at the same time. This is achieved by using the coordinate $q_{2}$ as a time variable. It is a classical result that in terms of the ' $q_{2}$ ' time variable, this system still has Hamiltonian form (see e.g. Ref. $3 \S 141$ ). Upon setting $q_{2}=\tau$, dropping the subscripts ' 1 ' on $q_{1}$ and $p_{1}$, the Hamiltonian in the new coordinates becomes $H=H(q, p, \tau)$, where we suppress the dependence on the fixed value of $E$. Thus we have reduced the order of the problem, but at the expense of obtaining explicit time dependence in the Hamiltonian. Taking the return time of the disk of trajectories to be $T$, a little thought yields the periodicity condition $H(q, p, \tau)=H(q, p, \tau+T)$. Systems like this are said to have one and a half degrees of freedom, the half accounting for the periodic time dependence. Thus we have arrived at the following conclusion:

\section{Hamiltonian systems of one and a half or two degrees of freedom are (at least locally) area preserving maps of a planar region.}

Maps like these are sometimes called symplectic maps.

Now let us turn to magnetic field lines. In particular, consider the arrangement of two current sources depicted in Fig. 2. This arrangement has a large current with density $J_{z}$ flowing in the $\widehat{z}$-direction and a smaller closed current with density $J_{a}$ that lies in the plane perpenducular to $\widehat{z}$ and is on average azimuthal. The separate magnetic field lines corresponding to the two currents are also depicted in this figure. It is not difficult to show by analyzing the cylindrical coordinate field line equations, 


$$
\frac{d z}{B_{z}}=\frac{d \rho}{B_{\rho}}=\frac{\rho d \phi}{B_{\phi}},
$$

that the field lines due to the total current, i.e. those of the superposition of the two fields depicted, are typically not closed and typically do not go to infinity.

Consider the symmetrical situation where the currents are concentrated to single wires, a vertical one along the $z$-axis corresponding to $J_{z}$ and a circular one that points in the $\widehat{\phi}$-direction and is located at $\rho=R, z=0$, corresponding to $J_{a}$. In order to simplify the discussion we suppose $J_{z}$ is much larger than $J_{a}$, and consider a region over which the azimuthal field produced by $J_{z}$ is approximately constant. The field lines can be described by a surface of section such as the one shown in Fig. 3. Here we have plotted points where the field lines pierce a given vertical plane containing the vertical wire, e.g. the plane $\phi=0$. The ordinate of this figure is $p:=r^{2} / 2$, where $r$ is a radius measured from the circular wire, and the abscissa $\theta$ is an angle measured around the circular wire. Thus, $(r, \theta)$ constitute a polar coordinate system located in any $\phi=$ constant plane. From the figure, it can be inferred that field lines wind around in a helical manner and lie on nested tori centered on the circular wire. Most field lines densely cover a torus, but some are indeed closed. Field lines that look like this are called integrable.

The two-current system described above is a sort of 'poor man's' version of the field lines that ideally occur in stellarator or tokamak fusion devices. In practice symmetry is necessarily broken and the field lines look more like those shown in Fig. 4. Observe that some appear to wander chaotically while others lie on tori.

So, what does $\nabla \cdot \mathbf{B}=0$ imply? The answer is that the map from the $p-\theta$ plane to itself that is defined by the field lines is an area preserving map, just like that described above for Hamiltonian systems. The field line picture is identical to the Hamiltonian trajectory picture with the role of time being played by the azimuthal coordinate $\phi$. Thus we have arrived at the following conclusion:

Magnetic field lines are (at least locally) trajectories of Hamiltonian systems of one and a half or two degrees of freedom. 
Thus magnetic field line maps are symplectic maps.

The relationship between magnetic field lines and Hamiltonian systems was recognized early on in the fusion program. The earliest reference I know of is that of Kruskal (Ref. 4) in 1952, who iterated an explicit area preserving map (similar to the standard map defined below) in order to describe the magnetic field of stellarators. Early papers in which field line Hamiltonians were obtained for integrable fields are Ref. 5 and Ref. 6. (See Ref. 7, which describes some of the ample contributions from the former Soviet Union.) Although many papers were written in the early 60's and 70's on this topic, a general and explicit Hamiltonian description for field lines does not appear to have been discovered until the early 1980's. Boozer (in Ref. 8) considered a potential representation for the divergence free magnetic field of the form $\mathbf{B}=\nabla \psi \times \nabla \theta+\nabla \phi \times \nabla \chi$, an idea that was used previously in less generality in plasma physics and dates way back to Euler. With this form for $\mathbf{B}$, the field line equations can be cast into the following Hamiltonian form:

$$
\frac{d \psi}{d \phi}=-\frac{\partial \chi}{\partial \theta}, \quad \frac{d \theta}{d \phi}=\frac{\partial \chi}{\partial \psi}
$$

where $\phi$ is the azimuthal angle, $\psi$ is a radial-like coordinate that plays the role of the momentum conjugate to $\theta$, and $\chi(\phi, \theta, \psi)$ is the Hamiltonian. This formulation has been widely used in stellarator research. (Seef also Ref. 9 pp. 8-10.) A more fundamental description was given by Cary and Littlejohn (Ref. 10) in terms of an action principle that depends on the vector potential $\mathbf{A}$,

$$
S[\mathbf{r}]=\int_{\mathbf{r}_{0}}^{\mathbf{r}_{1}} \mathbf{A} \cdot d \mathbf{r} .
$$

Here, as in Hamilton's principle of mechanics, the trajectories are pinned at the initial and final positions, $\mathbf{r}_{0}$ and $\mathbf{r}_{1}$, respectively. It is interesting to compare this action principle with the phase space action principle, the action principle of mechanics that directly gives Hamilton's equations

$$
S[q, p]=\int_{r_{0}}^{\mathbf{r}_{1}}(p \cdot d q-H d t) .
$$


If one singles out one of the coordinates in (4) to play the role of time, then comparison with (5) indicates that the corresponding component of the vector potential is associated with the Hamiltonian, while the other components are associated with the canonical momenta.

Lest one thinks the story is over, I mention the work of Mezic and Wiggins (Ref. 11) who give a description in terms of commuting vector fields that does not require the introduction of the vector potential.

\section{TWIST}

The twist condition is an ingredient that is used in many important theorems in Hamiltonian dynamics, theorems due to Arnold, Moser, Aubry, Mather, and others. The condition is used in theorems that apply to both the ordinary differential equation and map descriptions of Hamiltonian systems. Because of this, we will describe the twist condition below in both contexts. Ultimately, the prevalence of the twist condition can be traced to the form of the Hamiltonians that describe particle dynamics.

First, consider a one and a half degree-of-freedom system, i.e. one with a single $q$ and $p$ that satisfies $H(q, p, t)=(q, p, t+T)$. The Hamiltonians for a driven swing and for magnetic field lines without symmetry are of this type. For a particle system, the swing included, the Hamiltonian is the sum of kinetic and potential energy parts, $H=p^{2} /(2 m)+V(q$, $t)$, with $V(q, t)=V(q, t+T)$. Since $m \dot{q}=p$ it is evident that larger canonical momentum implies larger velocity, a condition that is true for many Hamiltonians but obviously not all. This simple monotonicity condition is the essence of the twist condition.

More generally, consider near-integrable systems with one and a half degrees of freedom, which have Hamiltonians of the form $H=H_{0}(J)+\epsilon H_{1}(\theta, J, t)$ with $\epsilon<<1$. Here the variables $(\theta, J)$ are assumed to be action-angle variables for an integrable system with Hamiltonian $H_{0}(J)$. The frequency is defined by $\omega_{0}(J):=\partial H_{0} / \partial J$ and the twist condition for this class of system is given by

$$
\frac{\partial \omega_{0}(J)}{\partial J}=\frac{\partial^{2} H_{0}(J)}{\partial J^{2}}>c>0 \text {. }
$$


where $c$ is a real number. (Note, the twist condition exists if $\partial \omega_{0}(J) / \partial J<c<0$, which turns into (6) under time reversal.)

In Sec. II we saw how Hamiltonian systems and magnetic field line equations are related to symplectic maps. Such maps inherit the twist condition. Investigation of field lines (or Hamiltonian trajectories) conventionally requires numerical integration of differential equations like those of Eq. (2). However it is now understood that Hamiltonian systems have universal behavior that is captured by studying explicit symplectic maps of the plane.

The most studied symplectic map is the standard map (sometimes called the ChirikovTaylor map), which is given by

$$
y_{n+1}=y_{n}-\frac{k}{2 \pi} \sin (2 \pi x), \quad x_{n+1}=x_{n}+y_{n+1}
$$

Here $y$ is a momentum-like variable and $x$ is coordinate-like variable that is assumed to be periodic with period 1 . Sometimes we use $z:=(x, y)$ and write $(7)$ compactly as $z_{n+1}=$ $T\left(z_{n}\right)$.

Figures 3 and 4 of Sec. II were generated by repeated iteration of the standard map. In Fig. 3 some of the horizontal lines are filled in while some are merely isolated points. These are, respectively, the rational and irrational tori of the magnetic field configuration. The rational tori are composed of periodic orbits, which satisfy $z=T^{n}(z)=T \circ T \circ T \ldots T(z)$, where $\circ$ means composition of functions (i.e. $T^{n}(z)$ denotes the quantity obtained upon inserting the map inside itself $n$ times). If after iterating the map $n$-times one returns to the same point, then one has a periodic orbit of period $n$. On the other hand, irrational tori are densely fill-out upon repeated iteration. An important quantity is the rotation number, $\omega$, which is defined to be the average horizontal jump per iteration:

$$
\omega:=\lim _{n \rightarrow \infty} \frac{x_{n}}{n}
$$

Here, one suspends the periodic boundary condition on $x$ when taking the limit. For both rational and irrational tori the rotation number exists, and is correspondingly a rational and irrational number. The safety factor, $q:=2 \pi / \omega$, is conventionally used in fusion physics, 
and it measures the mean ratio of the number of turns the long way around the torus to the short way around. In Fig. 4 we see some irrational tori (or invariant tori as they are often called) and remnants of the rational tori as isolated periodic orbits. Periodic orbits play a large role in what is to come in Sec. VI.

Now we define the twist condition for maps: a map satisfies the twist condition (is a twist map) if points higher up along a vertical line make larger jumps in the horizontal direction. This will be true if $\partial y_{n+1} / \partial x_{n}>c>0$. Thus we expect the rotation number (when it exists) to increase monotonically in the vertical direction. This is indeed the case for the standard map, which is the prototype twist map.

\section{NONTWIST}

So, what is nontwist? Quite simply, a nontwist system is any system that does not satisfy the twist condition. There are many ways that this can happen. For example, a system can have no twist, which in the differential equations context means $\partial \omega_{0}(J) / \partial J \equiv 0$, while in the map context means $\partial y_{n+1} / \partial x_{n} \equiv 0$. Alternatively, there can be a single point at which these quantities vanish, and the vanishing can be of arbitrarily high order. The most important violation of the nontwist condition is that for which there is a single simple zero; i.e. for which $\omega_{0}(J)$ possesses a simple maximum or minimum or for which the rotation number of the map has a simple maximum or minimum. Universal behavior of this kind of system is captured by a map that we have called ${ }^{12}$ the standard nontwist map, which is defined by

$$
y_{n+1}=y_{n}+b \sin \left(2 \pi x_{n}\right), \quad x_{n+1}=x_{n}+a\left(1-y_{n+1}^{2}\right)
$$

where $a$ and $b$ are parameters and again $x$ has period 1 . There are other ways of writing nontwist maps, but all of those with the simple extremum property are essentially the same map, because they can be transformed into this form by means of a coordinate transformation. Note, that no coordinate transformation can eliminate the two parameter dependence. We have studied the standard nontwist map in great detail; some of our work will be described in Secs. V, VI, and VII. 
The nontwist map possesses a shearless curve, a curve along which the twist condition is violated. It is easy to define this quantity when $b=0$; for this case, the standard nontwist map reduces to an integrable map, like that of Fig. 3, for which all the orbits lie on horizontal lines. Evidently, the twist condition is violated along the horizontal line $y=0$. For $b \neq 0$ it is a greater challenge to define the shearless curve, one we meet in Sec. VI.

In spite of the fact that prior to this decade nontwist systems received little attention, they occur in many physical systems. Below we describe several of these. In IV A we discuss how nontwist must occur in atmospheric and laboratory zonal flows, where we began studying this phenomena about ten years ago. In IV B we discuss nontwist in the magnetic field line context, and in IV C we briefly mention several other physical problems where nontwist systems occur.

\section{A. Zonal flows and chaotic advection}

Zonal flows are azimuthal jets that occur in planetary atmospheres. The jet stream and polar night jet are examples that occur in the earth's atmosphere, and the great red spot is intimately related to zonal flows in Jupiter's atmosphere. Because of rotation, these flows are predominately two-dimensional with the altitude being the ignorable dimension. To leading order the flows are directed along lines of latitude, either eastward or westward, with variation in the flow speed along lines of longitude. This variation possesses a maximum and the flows tend to be confined in longitude, which is why they are called jets or zonal flows. Because of the maximum in flow speed, zonal flows give rise to transport phenomena, including barriers, described by nontwist Hamiltonian systems. ${ }^{12-14}$ The maximum in the flow profile corresponds to the shearless curve.

There is a long history of rotating fluid laboratory experiments (see e.g. Ref. 15) designed to simulate facets of atmospheric dynamics. Our interest in the subject was captured by an experiment in the laboratory of Swinney ${ }^{16}$ (see also Refs. 17-19) and in particular the connection between drift wave transport and the fluid mechanics of zonal flows that this 
experiment simulates. Drift waves are described to leading order by the Hasegawa-Mima equation and the same is true for the fluid dynamics of the experiment. The experiment is equipped with sophisticated particle tracking capability, which is ideal for measuring the transport of tracer particles that are added to the fluid.

The tracer particles are to high accuracy governed by a Hamiltonian system of differential equations. This is because the velocity field is nearly two-dimensional and satisfies $\nabla \cdot \mathbf{v}=0$, and because the tracer particles move with fluid. These conditions imply the following equations for the trajectories of the tracer particles:

$$
\dot{x}=v_{x}=\frac{\partial \psi}{\partial y}, \quad \dot{y}=v_{y}=-\frac{\partial \psi}{\partial x}
$$

where $\psi$, the streamfunction, is the Hamiltonian, and the coordinates $(x, y)$ are the canonical coordinates. They can be viewed as longitude and latitude or as azimuthal and radial coordinates in slab approximation. Thus a 'pure' zonal flow occurs if $\psi=\psi(x)$ and $v_{y}(x)$ is nonmonotonic (with an extremum), and therefore flows that are perturbations of this flow will violate the twist condition.

Experimentally it was observed ${ }^{18}$ and in simplified models shown ${ }^{13,14}$ that nonmonotonic velocity flow creates a strong transport barrier that is located in the region where the velocity profile attains its maximum, i.e. near the shearless curve. The reason for this is twofold:

(i) Because of the maximum in velocity, it can be shown that generally the density of low order resonances (island chains) decreases as one approaches the shearless curve. Typically higher order resonances are smaller and produce less damage.

(ii) In the fluid mechanical and in some plasma models (see below) perturbations of the pure zonal flow by the superposition of eigenmodes produces resonances that are bounded away from the shearless curve. (That they are bounded away is a consequence of the physics of the linear theories.) Thus the eigenmodes must have large amplitudes (or strong turbulence is needed) to overlap and thereby destroy the shearless invariant torus. 
In plasmas the zonal flow problem is analogous to $\mathbf{E} \times \mathbf{B}$ transport, with the electrostatic potential being the Hamiltonian. Accordingly, if the radial electric field is not monotonic, transport in the plane perpendicular to the magnetic field can be modeled with an area preserving map that violates the twist condition. Nonmonotonic radial electric fields are believed to be present in the tokamak edge when there is good confinement and we have recently found the same to be true in nontwist map models. ${ }^{20}$

\section{B. Nonmonotonic $q$-profiles}

As described in Sec. II magnetic field lines in toroidal plasma devices, such as tokamaks and stellerators, ideally lie on and wrap helically around nested tori. Also recall that the $q$-profile is the average pitch of this wrapping. Thus nonmonotonic $q$-profiles correspond to nontwist maps. In experiments it has been observed that nonmonotonic $q$-profiles are correlated with enhanced confinement. ${ }^{21}$ The map models obtained in Ref. 20 contain this effect, as well as that of the nonmonotonic radial electric fields described above, and the contribution of each to transport was analyzed. We refer the interested reader to this reference for details.

\section{Other physical systems}

Nontwist maps arise in many other physical problems. In celestial mechanics, when planetary gravitational potentials are not spherically symmetric because of oblateness of the planets, there are corrections to the Keplerian orbits that are governed in essence by a nontwist map. ${ }^{22}$ Other problems described by nontwist maps include the dynamics of rays in a cylindrical waveguide with a periodic array of lenses, ${ }^{23}$ particle accelerators with

nonmonotonic tune, ${ }^{24}$ and SQUID's and polymers. ${ }^{25}$ 


\section{RECONNECTION IN NONTWIST SYSTEMS}

Magnetic field line systems that do not satisfy the twist condition have a richer Hamiltonian structure than those that do not. In Figs. 4 and 5a we see the usual island structure that occurs in Hamiltonian systems with the twist condition, the usual island structure of tokamak and stellarator geometries. However, given a set of fixed points, the $\times$-points and 0-points of the figure, there are two topologies, i.e. there are two ways to hook up the separatrices. The alternative way is shown in Figs. 5b and 5c. The phenomenon where the separatrices change from one of these topologies to the other is called reconnection, and it is an example of what is called a global bifurcation.

Reconnection of this type was noticed early-on in the magnetic fusion context where it was suggested as an explanation for rapid current penetration in tokamaks. ${ }^{26} \mathrm{~A}$ discussion in the context of Hamiltonian systems was given in Ref. 27. We first pointed out that this kind of reconnection is a consequence of violation of the twist condition in Refs. 12, 13, and 14 and discussed it in subsequent work (Ref. 28). Several other contributions have been

made in a variety of contexts, ${ }^{29-32}$ too many to describe in detail here. Instead we present a picture gallery of reconnection phenomena in the standard nontwist map in Figs. 6 and 7 . In these figures the parameters $a$ and $b$ are chosen so that the map is nearly integrable, as opposed to the values chosen for Fig. 8, which contains both topologies nestled together with chaotic trajectories. Upon magnification of this figure one sees both topologies intertwined on ever finer scales. This is a harbinger of a difficulty associated with finding periodic orbits that is addressed in the next section.

\section{LAST TORUS OF THE STANDARD NONTWIST MAP}

In order to understand transport, one must understand how and when invariant tori break. In Fig. 3 we see that trajectories, being bound to horizontal lines, the invariant tori, cannot migrate in the vertical direction, while in Fig. 4 tori are broken and migration can 
occur. Thus we turn to our main problem:

For which values of the standard nontwist map parameters $a$ and $b$ is the shearless curve with rotation number equal to the inverse of the golden mean critical?

Recall the rotation number, $\omega$, is the average jump per iteration (suspending periodicity) in the horizontal direction, $\omega=\lim _{n \rightarrow \infty} x_{n} / n$, and the shearless curve is the curve along which the twist condition fails.

One could attempt to solve the main problem by brute force numerical iteration of the standard nontwist map. With careful numerics this procedure can give a sense of the $a-b$ parameter space and it might be sufficient to answer some engineering questions, but besides being inelegant, such a technique is limited in accuracy and gives limited insight into how tori break. In contrast, for twist maps there is a criterion due to Greene that allows one to perform extremely accurate computations and to understand the self-similar nature of phase space near a torus that is on the verge of breaking. For this reason we attack the main problem (in Sec. VIC) by extending Greene's criterion to nontwist maps. This extension is nontrivial and so we briefly review Greene's criterion in the context of twist maps in Sec. VIA, in preparation for the generalization to nontwist maps that we implement in Sec. VIC. Since Greene's criterion uses periodic orbits, we describe in Sec. VIB how the involution decomposition that arises from discrete symmetries can be used to find them. For $b=0$, the shearless curve corresponds to the line $y=0$, but for $b \neq 0$, as noted above, some care is required to make precise what is meant by this quantity. We discuss this in Sec. VIC where we describe our solution to the main problem.

\section{A. Greene's criterion}

Greene's criterion is a method for determining parameter values for the destruction of invariant tori in twist maps. Unlike KAM theory, these tori are far from the integrable limit. Since physical systems are often not nearly integrable, this procedure is of greater 
importance, but one must resort to numerics to implement it. Greene originally calculated the parameter value for the destruction of the last invariant torus in the standard map.

Green's calculation has two essential ideas. The first idea is that one can approximate an invariant torus by a sequence of periodic orbits. It is evident from Fig. 3 that this is possible for integrable systems, since this picture depicts both rational and irrational tori, but indeed it was generated on a computer screen for which all orbits are periodic. (Clearly there are a finite number of pixels on any screen or in any data array.) When a system is not integrable, it remains true that near an invariant torus are periodic orbits of arbitrarily high order.

As one increases the parameter $k$ in the standard map more and more invariant tori break. A body of research leading up to and including the proof of the KAM theorem suggested that sturdy tori are those with winding numbers that are difficult to approximate by rational numbers. A class of numbers called noble numbers, a class that includes the golden mean and its inverse, are especially difficult to approximate. For this reason Greene conjectured that the last surviving invariant torus has a rotation number equal to the inverse of the golden mean,

$$
\frac{1}{\gamma}=\frac{\sqrt{5}-1}{2}=\frac{1}{1+\frac{1}{1+\ldots}}=0.618 \ldots,
$$

and he calculated the value of $k$ for which this torus is critical to very high precision. He did this by finding a particular sequence of periodic orbits with rotation numbers, $\omega_{i}=n_{i} / m_{i}$ ( $n_{i}$ and $m_{i}$ are integers) that limit to $1 / \gamma$. The $\omega_{i}$ 's he used were obtained by truncating the continued fraction expansion and writing the result as a simple fraction. This gives $\omega_{i}=F_{i} / F_{i+1}$, where the $F_{i}$ 's are the Fibonacci numbers, $1,1,2,3,5, \ldots$.

The second essential idea concerns the stability of the periodic orbits. In the vicinity of stable periodic orbits, nearby orbits remain nearby, while unstable orbits move away exponentially. The type of periodic orbit depends upon the residue which Greene defined by

$$
R:=\frac{1}{4}\left[2-\operatorname{trace}\left(D T^{n}\right)\right] .
$$


where $D T^{n}$ is a deceptively simple notation for the matrix obtained upon linearizing $T^{n}$ about the periodic orbit. If $0<R<1$ the periodic orbit is stable or elliptic, if $0<R$ or $R>1$ the periodic orbit is unstable or hyperbolic, and if $R=0$ or $R=1$, the periodic orbit is parabolic, which is characteristic of periodic orbits in integrable systems. Greene calculated the residues for the periodic orbit sequence that limits to the golden mean and observed that if $\lim _{i \rightarrow \infty} R_{i}=0$, then the torus with rotation number $1 / \gamma$ exists, while if $\lim _{i \rightarrow \infty} R_{i}$ diverges then the torus does not exist. At criticality he found $\lim _{i \rightarrow \infty} R_{i} \approx .25$. He was able to creep in on the critical value of the parameter by examining the residues, and in this way obtain $k_{c} \approx .97 \ldots$ to a gazillion places.

\section{B. Involution decomposition and periodic orbits}

In order to implement Greene's method it is necessary to find periodic orbits. An efficient way to do this is to exploit discrete symmetries possessed by maps of interest. In traditional course work we learn about Noether's theorem, and how it relates symmetries to constants of motion. However, the symmetries involved in this theorem are continuous symmetries, symmetries such as space translation that depend continuously on a parameter. Although discrete symmetries are not useful for obtaining constants of motion, it was recognized by Birkhoff (see e.g. Ref. 33) and de Vogeleare (Ref. 34) that they do organize the periodic orbit structure of Hamiltonian systems and, most importantly, it was shown by Greene (Ref. 35) how they can be used in numerical computation to vastly expedite the search for periodic orbits in maps.

The most well-known discrete symmetry is time reversal symmetry, where the equations of motion are invariant under $t \rightarrow-t$ and $p \rightarrow-p$. Hamiltonian systems do not always possess this time reversal symmetry. In particular, the nontwist systems of interest here do not satisfy $H(q, p) \neq H(q,-p)$. However, another discrete symmetry exists that amounts to $t \rightarrow-t$ and $q \rightarrow-q$, and we exploit this discrete symmetry to obtain periodic orbits for the standard nontwist map. 
The story of discrete symmetries and their association with periodic orbits is a long one, so we only touch on two salient points and direct the reader to Ref. 28 where we review the method and apply it to the standard nontwist map. The two points are

(i) Discrete symmetries in Hamiltonian differential equations are manifest in the corresponding area preserving maps as involution decompositions. If we represent maps such as those of (7) and (9) as $z_{n+1}=T\left(z_{n}\right)$, then we have an involution decomposition if $T=I_{1} \circ I_{0}$, where $I_{1} \circ I_{1}=i d e n t i t y$ and $I_{0} \circ I_{0}=i d e n t i t y$. (Recall here $\circ$ means composition of functions.)

(ii) Periodic orbits can be found by searching symmetry lines, which are curves in the plane that map into themselves under $I_{1}$ or $I_{0}$.

The second point above is most important because it reduces the search for periodic orbits to a one-dimensional root finding problem. For the standard nontwist map the symmetry lines are shown in Fig. 9. By searching along these lines we have been able to calculate periodic orbits of very high order to high accuracy.

\section{Results}

Now we are in a position to solve our main problem. However, there is a difficulty. In Greene's original calculation he was able to find the necessary periodic orbits by searching a particular symmetry line. In the present case it was discovered that periodic orbits with rotation numbers $\omega_{i}=F_{i} / F_{i+1}$ sometimes do not exist and sometimes come in pairs. It is perhaps not too surprising that they could come in pairs, since a resonance will generally open two island chains at the two equal values of the $q$-profile. This is easy to show when $b=0$, but as $b$ increases the periodic orbits can collide and disappear all together. In Fig. 10 we show the behavior as $b$ is increased for $a=.618$ and rotation number $3 / 5$. At a critical value of $b$, between four and five, the orbits collide. Thus we have a big problem, because 
we do not know whether or not a given periodic orbits exists for values of the parameters $a$ and $b$.

To get a handle on this problem we introduced the notion of the $r / s$-bifurcation curve, which is defined to be the locus of points $(a, b)$ such that the periodic orbit with rotation number $r / s$ is at its collision point. We can write this curve as $b=\Phi_{r / s}(a)$. In practice we fix $a$ and then ease $b$ away from zero until we are at bifurcation. The $r / s$-bifurcation curves are useful because we know that below the curve the pair of periodic orbits exists, but above it they do not.

Evidently periodic orbits on the curve are at a point of degeneracy, and we call periodic orbits of this type shearless periodic orbits. Now we can define the shearless curve when $b \neq 0$ :

Shearless periodic orbits limit to the shearless curve.

In Fig. 11 we show several $r / s$-bifurcation curves that indicate the approach to a limiting curve. We call this limiting curve $\Phi_{1 / \gamma}$. Note this figure only shows periodic orbits with rotation numbers representative of half of the Fibonacci sequence. It turns out that periodic orbits with rotation numbers equal to only half of the sequence exist, but this is sufficient for effecting the limit of their residues as calculated from Eq. (12). However, there is another surprise: these residues do not limit to a number, but limit to a period-6 cycle; i.e. the sequence of residues has six convergent subsequences. This makes the calculations of convergence very difficult because one must work much harder to obtain the same level of convergence of the residue values as for the twist case. We overcame this difficulty by exploiting some tricks involving symmetry, and we refer the reader to Ref. 28 for details.

The upshot is that we calculated periodic orbits for $r / s=75,025 / 121,393$ and obtained the following critical values of the parameters:

$$
a \approx 0.686049, \quad b \approx 0.742497002412
$$

In Fig. 12 we show the standard nontwist map at these critical values. Here we see the last invariant torus bounding an orbits generated from a single initial condition. 


\section{RENORMALIZATION}

The jagged structure of the last invariant torus shown in Fig. 12 suggests a self-similar structure reminiscent of phase transitions. This suggestion is strengthened in Fig. 13 which is a blow-up and rescaling of a region of Fig. 12. The analogy to phase transitions is not merely suggestive, but there is a well-developed theory for renormalization in Hamiltonian systems. Early work on the theory was due to Kadanoff (Refs. 36 and 37), but the picture was completed by Greene and Mackay (Refs. 38, 39, 40, and 41). In this theory, as in critical

phenomena, there is a renormalization group operator, but here fixed points correspond to invariant tori at criticality rather than to phase transitions. In Ref. 42 we obtained a new fixed point for this operator. Further discussion is beyond the scope of this paper; the interested reader is referred to this reference.

\section{SUMMARY}

In this paper we have described how magnetic field lines typically behave, by identifying the equations that define them with Hamilton systems of one and a half degrees of freedom or equivalently area preserving maps of planar regions. This enabled us to use the vast lore of Hamiltonian dynamics theory to say some general things about their behavior. In particular, we observed that magnetic field lines generally do not close on themselves and when there is a lack of symmetry they possess chaotic behavior. We have discussed the twist condition in both the differential equation and map settings, and we traced its origin to particlelike Hamiltonians. We described nontwist systems and gave examples of representative physical systems, including the tracking of particles in zonal flows and magnetic field lines in reversed shear configurations. Some Hamiltonian lore was reviewed, including the definition of such quantities as periodic orbits, the rotation number, and the residue. Reconnection in nontwist systems was discussed. The the use of discrete symmetries for finding periodic orbits was described in the context of Greene's method for finding the last invariant torus 
in twist systems. The difficult generalization of Greene's method to nontwist systems was also described. A few brief comments were made about renormalization.

Our goal here has been to acquaint the reader with some of ideas in this area of Hamiltonian field line dynamics, and the contributions of plasma physicists. However, there is an enormous literature in this area and many topics have by necessity been omitted, particularly those on transport.

\section{Acknowledgments}

I would like to thank Jim Hanson for sharing with me his collection of papers on the Hamiltonian description of magnetic fields. This work was supported by the U.S. Dept. of Energy Contract No. DE-FG03-96ER-54346. 


\section{REFERENCES}

${ }^{1}$ D. del-Castillo-Negrete, Dynamics and Transport in Rotating Fluids and Transition to Chaos in Area Preserving Nontwist maps, Ph.D. thesis, The University of Texas, Austin, October, 1994.

${ }^{2}$ C.L. Siegel and J. K. Moser, Lectures on Celestial Mechanics (Springer, Berlin, 1971).

${ }^{3}$ E.T. Whittaker, A Treatise on the Analytical Dynamics of Particles and Rigid Bodies, fourth edition, (Cambridge, New York, 1937).

${ }^{4}$ M.D. Kruskal, Some Properties of Rotational Transforms, Project Matterhorn Report, NY0-998, PM-S-5, Princeton University Forrestal Research Center, (1952).

${ }^{5}$ D.W. Kerst, Plasma Physics (J. of Nuc. Energy, part C) 4, 253 (1962).

${ }^{6}$ I.M. Gelfand, M. I. Graev, N. M. Zueva, A. I. Morozov, and L. S. Solov'ev, Soviet Phys. (Tech. Phys.), 6, 852 (1962).

${ }^{7}$ A.I. Morozov and L.S. Solov'ev, in Reviews of Plasma Physics 2, ed M.A. Leontovich, (Consultants Bureau, Plenum, New York, New York 1966).

${ }^{8}$ A.H. Boozer, Phys. Fluids 24, 1999 (1981).

${ }^{9}$ A.H. Boozer, Plasma Confinement in Encyclopedia of Physical Science and Technology vol.13 (Academic Press, New York, 1992).

10 J.R. Cary and R.L. Littlejohn, Ann. Phys. 151, 1 (1983)

${ }^{11}$ I. Mezic and S. Wiggins, J. . Nonlinear Science 4, 157 (1994).

${ }^{12}$ D. del-Castillo-Negrete and P.J. Morrison, "Magnetic Field Line Stochasticity and Reconnection in a Non-monotonic q-profile," Bull. Am. Phys. Soc. 37, 1543 (1992).

${ }^{13}$ D. del-Castillo-Negrete and P.J. Morrison, Hamiltonian Chaos and Transport in Quasigeostrophic Flows, Research Trends in Physics: Chaotic Dynamics and Transport in 
Fluids and Plasmas, eds. I. Prigogine, et al., (American Institute of Physics, New York, 1993)

${ }^{14}$ D. del-Castillo-Negrete and P.J. Morrison, Phys. Fluids A 5, 948 (1993).

${ }^{15}$ R. Hide, Philos. Trans. R. Soc. London A 250, 441 (1958).

${ }^{16}$ J. Sommeria, S.D. Meyers, and H.L. Swinney, Laboratory Model of a Planetary Eastward Jet, Nature 337, 58 (1989).

17 J. Sommeria, S.D. Meyers, and H.L. Swinney, Experiments on Vortices and Rossby Waves in Eastward and Westward Jets; Nonlinear Topics in Ocean Physics, ed. A. Osborne (North-Holland, Amsterdam, 1991).

${ }^{18}$ R.P. Behringer, S.D. Meyers and H.L. Swinney, Chaos and Mixing in a Geostrophic Flow, Phys. Fluids A 3, 5, 1243 (1991).

19 T.H. Solomon, W. Holloway, and H. Swinney, Shear Flow Instabilities and Rossby Waves in Barotropic Flow in a Rotating Annulus, submitted to Phys. Fluids A 5, 8 (1993).

${ }^{20}$ W. Horton, H.-B. Park, J.-M. Kwon, D. Strozzi, P.J. Morrison, and D.-.I Choi Phys. Plasmas 5, 3910 (1998).

${ }^{21}$ E. Mazzucato, S.H. Batha, M. Beer, M. Bell, R.E. Bell, R.V. Budny, C. Bush, T.S. Hahm, G.W. Hammett, F.M. Levinton, R. Nazikian, H. Park, G. Rewoldt, G.L. Schmidt, E.J. Synakowski, W.M. Tang, G. Taylor, and M.C. Zarnstorff, Phys. Rev. Lett, 77, 3145 (1996).

${ }^{22}$ W.T. Kyner, Mem. of the Am. Math. Soc. 81, 1 (1968).

${ }^{23}$ S.S. Abdullaev, CHAOS 4, 569 (1994).

${ }^{24}$ K.R. Symon and A.M. Sessler, in Proc. Int. Conf. on High-Energy Accelerators and Instrumentation (Centre Européen Pour La Recherche Nucleaire, Geneva, 1956) p. 44; A. Gerasimov, F.M. Israilev, J.L. Tennyson and A.B. Temnykh, Lecture Notes in Physics, 
Vol. 247 (Springer, Berlin, 1986) p. 154.

${ }^{25}$ S. M. Soskin, Phys. Rev. E 50, R44 (1994).

26 T.H. Stix, Phys. Rev. Lett. 36, 10 (1976).

27 J.E. Howard and S.M. Hohs, Phys. Rev. A 29, 418 (1984).

${ }^{28}$ D. del-Castillo-Negrete, J.M. Greene, and P.J. Morrison, Physica D 91, 1 (1996).

29 J. P. Van der Weele, T. P. Valkering, H. W. Capel and T. Post, Physica A 153, 283 (1988).

30 J. P. Van der Weele and T. P. Valkering, Physica A 169, 42 (1990).

${ }^{31}$ J. E. Howard and J. Humpherys, Physica D 80, 256 (1995).

${ }^{32}$ S. Shinohara and Y. Aizawa, Prog. Theo. Phys. 97, 379 (1997).

${ }^{33}$ G.D. Birkhoff, Dynamical Systems (American Mathematical Society, Colloq. Publ., revised 1966) Vol. 9.

${ }^{34}$ R. de Vogelaere, Contributions to the Theory of Nonlinear Oscillations, edited by S. Lefschetz (Princeton University Press, Princeton, New Jersey, 1958), Vol. IV., p. 53.

35 J.M. Greene, J. Math. Phys. 20, 1183 (1979).

${ }^{36}$ L.P. Kadanoff, Phys. Rev. Lett. 47, 1641 (1981).

37 S.J. Shenker and L.P. Kadanoff, J. Stat. Phys. 27, 631 (1982).

${ }^{38}$ J .M. Greene, Physica D, 18, 427 (1986).

39 J.M. Greene, in Chaos in Australia, edited by G. Brown and A. Opie, (World Scientific, Singapore, 1993) p. 8.

${ }^{40}$ R.S. MacKay, Renormalization in Area Preserving Maps, Thesis, Princeton 1982 (Univ. Microfilms Int., Ann Arbor MI).

${ }^{41}$ R.S. MacKay, Physica D 7, 283(1983). 
${ }^{42}$ D. del-Castillo Negrete, J.M. Greene, and P.J. Morrison, Physica D 100, 311 (1997). 


\section{FIGURE CAPTIONS}

FIG. 1. Depiction of Poincaré return map in a two degree-of-freedom Hamiltonian system.

FIG. 2. Depiction of a two-current system and magnetic field lines.

FIG. 3. Magnetic field line return map for a symmetrical two-current system.

FIG. 4. Magnetic field line return map for an unsymmetrical two-current system.

FIG. 5. Depiction of two possible separatrix possibilities, with transition point, in a nontwist system.

FIG. 6. A reconnection bifurcation in the standard nontwist map.

FIG. 7. Another reconnection bifurcation in the standard nontwist map.

FIG. 8. A depiction of the two possible separatrix possibilities together with chaos in the standard nontwist map.

FIG. 9. Symmetry lines for the standard nontwist map.

FIG. 10. Separation in $y$ and collision of a pair of periodic orbits in the standard nontwist map.

FIG. 11. Bifurcation curves limiting to $\Phi_{1 / \gamma}$.

FIG. 12. Standard nontwist map at criticality.

FIG. 13. Depiction of self-similar structure in the standard nontwist map at criticality. 